\title{
Broadly tunable multiple wavelength Brillouin fiber laser exploiting erbium amplification
}

\begin{abstract}
We experimentally demonstrate a highly stable and flawless-tuning-range multiple wavelength Brillouin fiber laser incorporating an erbium gain block. Free-running cavity modes that inherently circulate in the cavity of a Brillouin/erbium fiber laser, which limits wavelength tunability, are completely suppressed. At a Brillouin pump power of $2 \mathrm{~mW}$ and $130 \mathrm{~mW}$ of $1480 \mathrm{~nm}$ pump power, we obtained seven output channels. The tunability of the generated channels is only limited to $35 \mathrm{~nm}$ by the amplification bandwidth of the erbium gain used in the experiment. The first four channels have an output power each above $1.6 \mathrm{dBm}$ with the first and the seventh channels having a peak power of $8.19 \mathrm{dBm}$ and $-8.30 \mathrm{dBm}$.
\end{abstract}

